\title{
GAS INJECTION EXPERIMENT DURING ELETTRA OPERATION
}

\author{
E. Karantzoulis, J. Miertusova, F. Pradal, L. Tosi, G. Tromba, A. Vascotto \\ Sincrotrone Trieste ${ }^{\#}$, Italy
}

\section{Abstract}

Gas injection experiments have been performed to gain a better understanding of the effect of local vacuum conditions on beam lifetime and gas Bremsstrahlung. Various gases $\left(\mathrm{H}_{2}, \mathrm{~N}_{2}\right.$, Ar) were injected up to a pressure of $5 \mathrm{e}-6 \mathrm{mbar}$ into the storage ring during machine operations, with various currents, energies, ID gaps and machine fillings. Results of lifetime and gamma dose rate measurements at different pressure conditions are presented and discussed.

\section{LIFETIME DUE TO GAS DENSITY}

The vacuum system of the ELETTRA storage ring has been designed to guarantee long lifetimes of stored beams i.e. at least ten hours with $300 \mathrm{~mA}$ at $2 \mathrm{GeV}$. The total lifetime in an electron storage ring is mainly determined by: i) elastic and inelastic scattering on the residual gas molecules, ii) Touschek effect, i.e. scattering of electrons within the bunch, iii) trapping of charged particles in the beam potential and iv) quantum lifetime. In the ELETTRA synchrotron light source the total lifetime is Touschek dominated. For our purposes, the machine setting parameters, e.g. RF cavities temperatures, machine files, etc. are considered implicitly as constant parameters and are omitted in this work, as well as quantum effects which are only important for machines with extremely small apertures.

In this paper, we focus on lifetime measurements under different vacuum conditions. The higher the operating pressures the larger the cross sections of both the elastic and inelastic scattering and the probability of ion trapping can also increase in the presence of higher ion masses in the vacuum chamber.

The elastic scattering lifetime $\tau_{\mathrm{el}}$ is usually expressed as:

$$
\frac{1}{\tau_{\text {elastic }}} \sim \frac{Z^{2} P}{E^{2}}\left(<\beta_{x}>\frac{\beta_{x 0}}{A_{x 0}^{2}}+<\beta_{y}>\frac{\beta_{y 0}}{A_{y 0}^{2}}\right)
$$

where $\mathrm{E}$ is the beam energy, $\mathrm{P}$ is the pressure of a gas with atomic number $\mathrm{Z}$ around the ring, $\left\langle\beta_{\mathrm{x}, \mathrm{y}}\right\rangle$ the average $\beta$-functions around the ring and $A_{(x, y) 0}$ the limiting transverse half apertures.

${ }^{\#}$ URL: www.elettra.trieste.it
The gas pressure varies with the total beam current $I_{t}$ due to desorption effects as:

$$
<P>=<P_{O}>+<\frac{d P}{d I_{t}}>I_{t}
$$

where $\left\langle\mathrm{dP} / \mathrm{dI}_{\mathrm{t}}\right\rangle$ is the average gas desorption coefficient.

The inelastic beam-residual gas encounters give rise to Bremsstrahlung gamma rays causing a pressure dependent beam loss and the lifetime can be evaluated [2] by:

$$
\tau_{B}=\frac{X_{j}}{\underset{j}{c W \rho_{j}}}
$$

where $\mathrm{c}$ is the relativistic electron velocity and $\mathrm{W}$ is the probability of the electron being lost from the machine per radiation length $\mathrm{X}$ traversing a material with density $\rho_{\mathrm{j}}$. Both the radiation length as well as the material density can be expressed using the atomic weight $A$, the atomic number $\mathrm{Z}$ and the molecular partial pressures $\mathrm{P}_{\mathrm{i}}$ of the gas present in the vacuum chamber. The gas composition used to be represented by a hypothetical monatomic gas with $Z=4$ to 5 . As can be seen, to calculate the beam lifetime due to the gas density, the average values of pressures, atomic numbers, etc. are used to characterize the vacuum conditions in the chamber. However, the storage ring vacuum system is equipped with pumps located at a regular distance from each other and the pressure presents a parabolic distribution with minima at the pump location and the term average pressure is only used to evaluate the pressure between these two extremes. From the vacuum point of view, the operating pressure $\mathrm{P}$ in the ring is given by:

$$
P=\frac{Q_{T}}{S}
$$

where $\mathrm{QT}_{\mathrm{T}}$ is the total gas load which is the sum of gas loads due to leakage $\left(\mathrm{Q}_{\mathrm{L}}\right)$, out-gassing $\left(\mathrm{QD}_{\mathrm{D}}\right)$, permeation $(\mathrm{QP})$ and induced desorption (QI). The pumping speed $\mathrm{S}$ is a dynamic parameter which varies with pressure. Towards lower pressures the pumping speed exponentially diminishes in time and the real, or so called "effective" pumping speed (assuming the proper conductance of the pump's connection to the vacuum chamber) $S_{\text {ef }}$ has to be taken into account [3]. All these vacuum parameters determining the operating pressure in the ring are strictly local values. The thermal and induced desorption as well as the permeation depends on the local degree of cleanliness and on the material quality of the vacuum chamber walls. If the storage ring is not perfectly tight the gas load from leakage is also rigorously localized. 
The pressure in the ELETTRA storage ring is monitored by means of sputter-ion pump (SIPs) pressure readings [4]. In each of the $40 \mathrm{~m}$ long vacuum sectors, 25 SIPs are installed. Pressure profiles in all six vacuum sectors are displayed on the vacuum panels in the control room. From the pressure values $\mathrm{P}_{\mathrm{i}}(\mathrm{N}(=150)$ is the total number of pumps) the average pressure is calculated as:

$$
<P>=\sum_{i=1}^{N} P_{i} / N
$$

Equation 5 is only an approximation and does not always reflect accurately the real vacuum conditions and to some extent the observed lifetime behavior. For example, in the case of a vacuum leak, a local pressure exceeding the normal operating pressure level by 2 to 3 orders of magnitude can be produced. In such cases a lifetime decrease is usually noticeable (a variation of about $30 \%$ less in most cases). However, the average pressure given from eq. 5 is affected only slightly and does not appear to fully explain the observed lifetime. To understand better the role of local pressure on the beam lifetime due to gas density the following experimental apparatus has therefore been installed in the ELETTRA.

\section{EXPERIMENTAL APPARATUS}

The drawing of the gas injection system allowing a local pressure increase in the storage ring is shown in fig. 1:

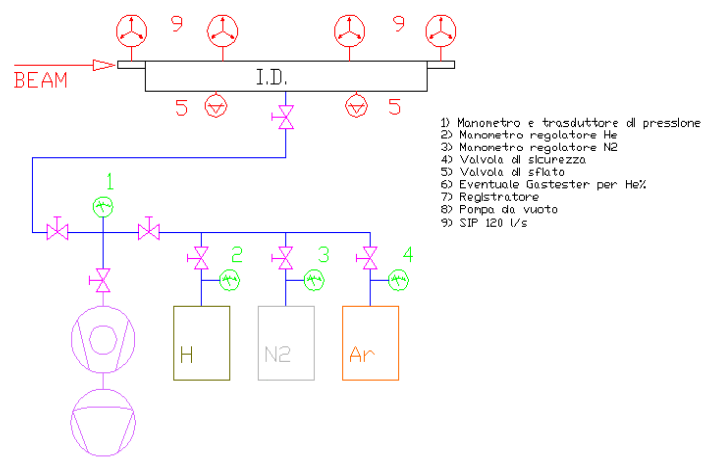

Fig. 1 - Experimental arrangement allowing gas injection into the ELETTRA storage ring

It is possible to inject through the dosing valve different gases $\left(\mathrm{H}_{2}, \mathrm{~N}_{2}\right.$, Ar) into the insertion device vacuum chamber ID_S3 and to create a local "pressure bump" up to 8e-6 mbar. In Fig. 2 many such bumps are shown obtained by regulating the gas flow. The average pressure values monitored during all the described experiments never exceeded 6e-9 mbar.

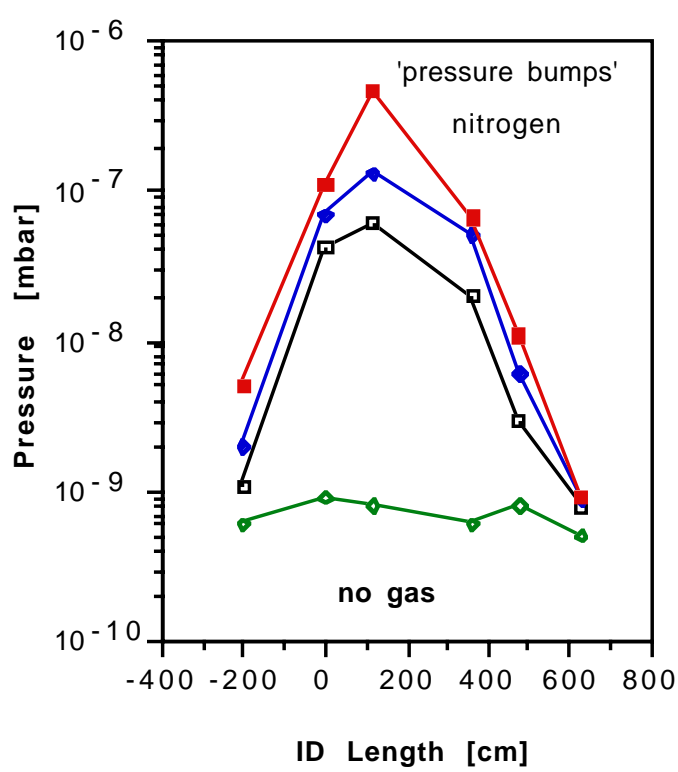

Fig. 2 - Pressure bumps created by nitrogen injection

\section{RESULTS AND DISCUSSION}

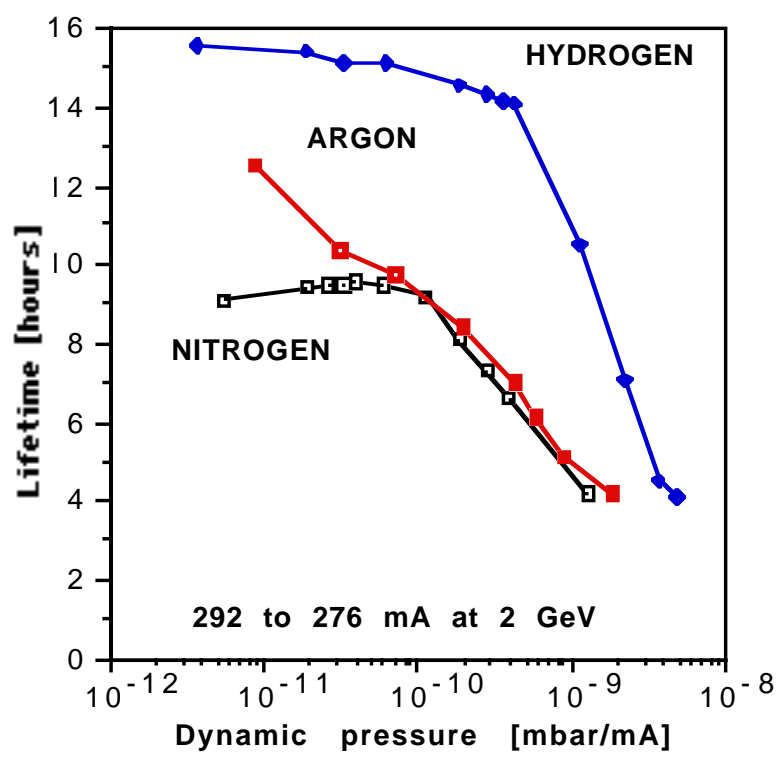

Fig. 3 - Total lifetime decrease caused by local dynamic pressure increase

Fig. 3 clearly shows that in the case of Ar, a minimum local pressure increase leads to an immediate lifetime reduction. This effect is less pronounced injecting nitrogen but at dynamic pressures higher than $1 \mathrm{e}-10 \mathrm{mbar} / \mathrm{mA}$ the lifetime decreases are the same for both argon and nitrogen. On the other hand the hydrogen 'pressure bump' causes a negligible lifetime decrease up to a local dynamic pressure of $1 \mathrm{e}-9 \mathrm{mbar} / \mathrm{mA}$. It has been also observed that, switching off the SIPs, a uniform operating pressure varying from $3 e-9$ up to $7 \mathrm{e}-9$ mbar was created in the 
whole ring without any measurable change in lifetime whereas at the same average pressure (as defined in eq. 5) of $5 \mathrm{e}-9$ mbar with the pressure bump of nitrogen, the beam lifetime changed by as much as $30 \%(250 \mathrm{~mA}$ at 2 $\mathrm{GeV}$ ).

Since the pressure is measured only at certain points of the ring with an accuracy that is increasing with pressure, expression (1) should be used with care bearing in mind that it is valid for homogeneous pressures around the ring. Thus for large deviations from homogeneity it may be preferable to use the dominant gas species with the highest local pressure for the estimations of elastic scattering lifetime. Additionally eq. (1) does not take into account the effect of local partial pressures of out-gassing species and subsequently local desorption coefficients.

During the gas injection experiments, bremsstrahlung gamma rays measurements have been performed for radioprotection purposes in a non restricted area outside the shielding wall.

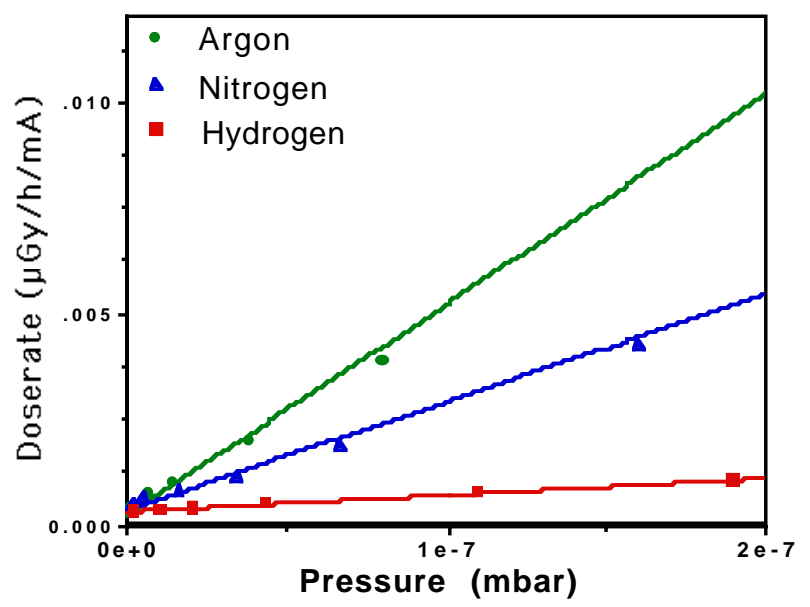

Fig. 4 - Gamma dose rate versus pressure

One of the environmental ionization chambers, used to monitor radiation levels around the beam-lines, allowed us to evaluate how different conditions of the vacuum in the chamber produced relevant changes in the bremsstrahlung radiation that is channeled into the beam-line during operation with the beam.

The gamma monitor is placed at about $3 \mathrm{~m}$ from the beam-line hutch in a position aligned with the straight section. It detects the secondaries generated by the bremsstrahlung gamma rays after interacting with the first optic component of the beam-line, such as the first mirror, and after the attenuation due to the lead shielding wall placed in the beam-line hutch.

The plots of gamma dose rates normalized per $\mathrm{mA}$ of stored current as a function of the local pressures in the chamber obtained with $\mathrm{Ar}, \mathrm{N}_{2}$ and $\mathrm{H}_{2}$ injections, are shown in fig. 4. Fits indicate a linear dependence according to:

$$
G=G_{0}+c Z P
$$

with $\mathrm{G}_{0}=3 . \mathrm{e}-4 \mu \mathrm{Gy} / \mathrm{h} / \mathrm{mA}$ and $\mathrm{C} \sim 3.5 \mathrm{e}+3 \mu \mathrm{Gy} / \mathrm{h} / \mathrm{mA} / \mathrm{mbar}$ as expected [5]. On the contrary, the gasses injected did not affect the bremsstrahlung gamma rays emission detected by a reference ionization chamber installed at the end of another straight section of the ring.

Data showed that any local increase of pressure in the straight section chamber, although not preventing beam injection and storage, can cause, from the radio-protection point of view, a significant increase of radiation levels outside the shielding around the corresponding beam-line exit.

Studies of ion trapping during the Gas Injection Experiments (GIE) have been reported elsewhere [6]. Here it is enough to mention that observations of beam characteristics were carried out for various beam configurations, currents and energies. The increase in vertical beam size with beam current that occurs only with high local pressure and $100 \%$ filling and which is large at low energy, is clearly evidence of ion trapping.

\section{CONCLUSIONS}

The Gas Injection Experiments at ELETTRA are very useful not only because they help in understanding the validity of approximations used but also because they reveal valuable information on the behavior of vacuum chambers having a higher pressure profile. New straight section vacuum chambers made from extruded aluminum are to be installed and these studies will highly help us to better analyze any future complications.

\section{REFERENCES}

[1] J. LeDuff, "Current and Density Limitations in Existing Electron Storage Rings", Nucl. Instrum. Methods A239,83 (1985)

[2] C. Benvenuti, R. Calder, O. Grobner, "Vacuum for Part. Accel. and Storage Rings", CERN-LEP-VA/87-66

[3] J. Miertusova, "New Asp. on the Vac. Syst. Design for the Future Synchr. Light Sources", Vacuum, 48, 7-9, p.751

[4] F. Giacuzzo, J. Miertusova, "Total Pressure Measurements", Proc. PAC'95, Dallas, U.S.A., 1995, p.684

[5] A. Rindi, "Gas bremsstrahlung from electron storage rings", Health Physics, Vol. 42, No. 2, 1982

[6] E. Karantzoulis et. al., "Collective Effects at ELETTRA", Proc. EPAC 1998, Stockholm, Sweden, 1998, p. 960 